\title{
Skeletal pattern in subjects with temporomandibular joint disorders
}

\author{
Oana Cristina Almășan¹, Mihaela Băciuțt2, Horea Artimoniu Almășan², Simion Bran², Liana Lascu1, \\ Mihaela lancu4 ${ }^{4}$ Grigore Băciuț ${ }^{3}$
}

\begin{abstract}
'Department of Prosthetic Dentistry, Faculty of Dentistry, University of Medicine and Pharmacy Iuliu Hațieganu, Cluj-Napoca, Romania

2Department of Maxillofacial Surgery and Implantology, Faculty of Dentistry, University of Medicine and Pharmacy Iuliu Hațieganu, Cluj-Napoca, Romania ${ }^{3}$ Department of Cranio-Maxillofacial Surgery, Faculty of Dentistry, University of Medicine and Pharmacy Iuliu Hațieganu, Cluj-Napoca, Romania ${ }^{4}$ Department of Medical Informatics and Biostatistics, Faculty of Dentistry, University of Medicine and Pharmacy Iuliu Hațieganu, Cluj-Napoca, Romania
\end{abstract}

Submitted: 2 February 2011

Accepted: 11 May 2011

Arch Med Sci 2013; 9, 1: 118-126

DOI: 10.5114 /aoms.2013.33072

Copyright (c) 2013 Termedia \& Banach

\section{Abstract}

Introduction: To establish the skeletal pattern in subjects with malocclusions and temporomandibular disorders (TMD); to assess the relationship between craniofacial skeletal structures and TMD in subjects with malocclusions.

Material and methods: Sixty-four subjects with malocclusions, over 18 years of age, were included in the study. Temporomandibular disorders were clinically assessed according to the Helkimo Anamnestic Index. Subjects underwent a lateral cephalogram. Subjects were grouped according to the sagittal skeletal pattern (ANB angle) into class I, II and III. Parametric Student tests with equal or unequal variations were used (variations were previously tested with Levene test). Results: Twenty-four patients with TMD (experimental sample); 40 patients without TMD (control group); interincisal angle was higher in class I and II $(p<0.05)$ experimental subjects; overjet was larger in experimental subjects; midline shift and Wits appraisal were broader in the experimental group in all three classes. In class III subjects, the SNB angle was higher in the experimental group $(p=0.01)$. Joint noises followed by reduced mandible mobility, muscular pain and temporomandibular joint (TMJ) pain were the most frequent symptoms in subjects with TMD and malocclusions.

Conclusions: Temporomandibular joint status is an important factor to consider when planning orthodontic treatment in patients with severe malocclusions; midline shift, large overjet and deep overbite have been associated with signs and symptoms of TMD.

Key words: temporomandibular joint disorder, malocclusion, lateral cephalometry.

\section{Introduction}

Temporomandibular joint disorder (TMD) is described as "a collective term embracing a number of clinical problems that involve the masticator musculature, the temporomandibular joint (TMJ) associated structures, or both" [1]. The etiology of TMJ disorders is poorly understood, but it is generally accepted that it is multifactorial, involving a large number of direct and indirect etiological factors, occlusion being frequently cited as one of the major etiological factors causing TMD [2]. Occlusal interferences,

\section{Corresponding author:}

Prof. Mihaela Băciuț

Department of Maxillofacial

Surgery and Implantology

Faculty of Dentistry University of Medicine and Pharmacy Iuliu Hațieganu Cluj-Napoca, Romania 37 Cardinal Iuliu Hossu Cluj-Napoca, Romania E-mail:mbaciut@yahoo.com, mbaciut@umfcluj.ro 
Angle Class II and III malocclusions, and large overjet have been associated with signs and symptoms of TMD [2, 3]. Malocclusion is widely accepted as being a cause of jaw dysfunction; in the literature there is contradictory evidence associating it with craniofacial symptoms [4-12]. The importance of occlusion and its role in perpetuating or causing TMD, compared with other factors, has been studied and is still debated nowadays [13, 14]. Subjects with malocclusions have been suggested to have a significantly higher prevalence of signs and symptoms of TMD than subjects without malocclusions [15]. Temporomandibular joint disorder has a multifactorial pathology, and it is difficult to demonstrate a direct correlation between one of the causes, for example occlusion, and TMD [16]. Endogenous and exogenous factors may disrupt the dynamic equilibrium (adaptive capacity) of the masticatory system, leading to the development and/or maintenance of TMD signs and symptoms [17]. Temporomandibular disorder, meaning the aberrant articular motion of the TMJ, can be induced by occlusion or malocclusion, untreated malocclusions, unstable occlusion, facial deformity, bruxism, estrogen level, anatomy, stress, nutrition, trauma, gender, parafunction, sleep disorders, posture, stress and other psychological factors $[18,19]$.

It is important to observe the temporomandibular disorder and the craniofacial relationship of patients with orthodontic treatment need from lateral cephalograms [20], as these investigations are routinely performed in order to show the craniofacial structures. No studies appear to have been performed on adult pre-orthodontic patients with malocclusions, where the signs and symptoms of manifest TMD have been diagnosed according to the Helkimo Anamnestic and Dysfunction Index and compared with an age- and gender-matched control group with malocclusions without any signs of TMD according to the Helkimo Anamnestic and Dysfunction Index.

The aims of this study were: (1) to evaluate the skeletal pattern of subjects with malocclusions and temporomandibular disorders (TMD) grouped by the sagittal skeletal relationship and to compare them to a sample of subjects with malocclusions without temporomandibular disorders, and (2) to determine existing associations between the TMJ status and the craniofacial morphology.

\section{Material and methods}

\section{Material}

A prospective, observational, analytic study was performed. A sample of 64 consecutive adult patients with malocclusions was included. All patients were referred to the Departments of Prosthetic Dentistry, Oral and Maxillofacial Surgery and Radiology at our university between 2008 and 2010 for ortho- dontic treatment. The inclusion criteria were: (1) age over 18 years, (2) no previous orthodontic treatment, (3) over 10 teeth in every arch, (4) no prosthesis. The exclusion criteria of the subjects were: (1) tumors in the joint region, (2) osteoarthritis of the TMJ, (3) osteoarthrosis of the TMJ, (4) traumatic injuries of the TMJ, (5) congenital craniofacial syndromes, (6) pregnancy, (7) periodontal disease, (8) acute infections and (9) acute dental problems. Temporomandibular joint function was clinically evaluated in each subject according to TMJ or muscle pain, joint sounds, and mouth opening limitations (Helkimo Anamnestic and Dysfunction Index). Based on these criteria, the patients were grouped in an experimental group which comprised subjects with TMD (Helkimo Index $>0$ ) and a control group which comprised subjects without TMD (Helkimo Index =0). The subjects were grouped by the sagittal skeletal relationship, according to ANB-angle differences into class I, II and III, with or without TMD, for assessing the relevant skeletal pattern according to the sagittal dimension. The structural relationships of the craniofacial and dental structures and the soft tissue relationships of subjects of the groups with TMD were compared with those of subjects without TMD according to their lateral cephalograms. Informed consent was obtained from each of the subjects before performing the study.

The procedures and protocol were approved by the institutional review board at the university and by the Ethics Committee, no. 173/26.10.2010.

\section{Methods}

A clinical examination of the TMJ, including joint and muscle palpation, mandible range of motion, joint noise analysis and evaluation of muscle or joint pain, was performed. The Helkimo Anamnestic and Dysfunction Index modified by Athanasiou and Melsen (HI) [21] was used for the clinical examination of the temporomandibular joint, evaluating mandible mobility, TMJ function and the existence of any muscular or joint pain and for the classification of the sample according to TMD presence and severity. The Helkimo Index used in the study, modified by Athanasiou and Melsen [21] for the clinical examination of the temporomandibular joint, evaluated: (1) the mandibular mobility (normal $=0$, reduced $=1$, severely reduced = 2); (2) the TMJ function (plane movement without sounds and deviations $<2 \mathrm{~mm}=0$, sounds in one or both joints and/or deviations $>2 \mathrm{~mm}=1$, locking or luxation = 5); (3) muscular pain (no pain on palpation $=0$; pain on palpation at one to three sites $=$ 1; pain at four or more palpation sites $=5$ ); (4) TMJ pain (no pain on palpation $=0$; lateral aspect pain $=$ 1 , distal aspect pain $=5$ ). . The sum of scores was used to evaluate the TMJ function as normal TMJ function (score 0), moderate TMD (score 1-4) and severe TMD (score 5-20) (Table I). 
Table I. The Helkimo Index modified by Athanasiou and Melsen [21] used for the TMJ clinical examination (sum of scores: 0 = normal temporomandibular joint function; 1-4 = moderate temporomandibular disorder; 5-20 = severe temporomandibular disorder)

\begin{tabular}{|c|c|c|c|}
\hline Criteria & Score $=0$ & Score $=1$ & Score $=5$ \\
\hline 1. Mandibular mobility & Normal & Reduced & Severely reduced \\
\hline $\begin{array}{l}\text { 2. Temporomandibular } \\
\text { joint function }\end{array}$ & $\begin{array}{l}\text { Plane movement without } \\
\text { sounds and deviations }\end{array}$ & $\begin{array}{l}\text { Sounds in one or both } \\
\text { joints and/or deviations }\end{array}$ & $\begin{array}{l}\text { Locking } \\
\text { or luxation }\end{array}$ \\
\hline 3. Muscular pain on palpation & No pain & Pain at $1-3$ sites & Pain at $4>$ sites \\
\hline 4. Temporomandibular joint pain on palpation & No pain & Lateral pain & Distal pain \\
\hline
\end{tabular}

A clinical examination of each subject was assessed in order to evaluate midline shift and associated anomalies of the subjects. Lateral cephalometry analysis was used to establish the skeletal pattern (Figure 1).

\section{Lateral cephalometric observations}

Lateral cephalograms of each patient were taken prior to diagnosis in centric occlusion, and with the Frankfort horizontal parallel to the floor at a magnification ratio of $1: 1$ and traced twice by two of the authors (AOC, AHA). All cephalometric radiographs were taken on the same radiographic device. Cephalograms were digitized; landmarks were identified using the method recommended by Steiner [22], Hasundd et al. [23], Segner and Hasund [24], Scheideman et al. [25]. The skeletal sagittal relationship was classified as, according to the ANB angle (difference between SNA angle - sella-nasion-A point and SNB

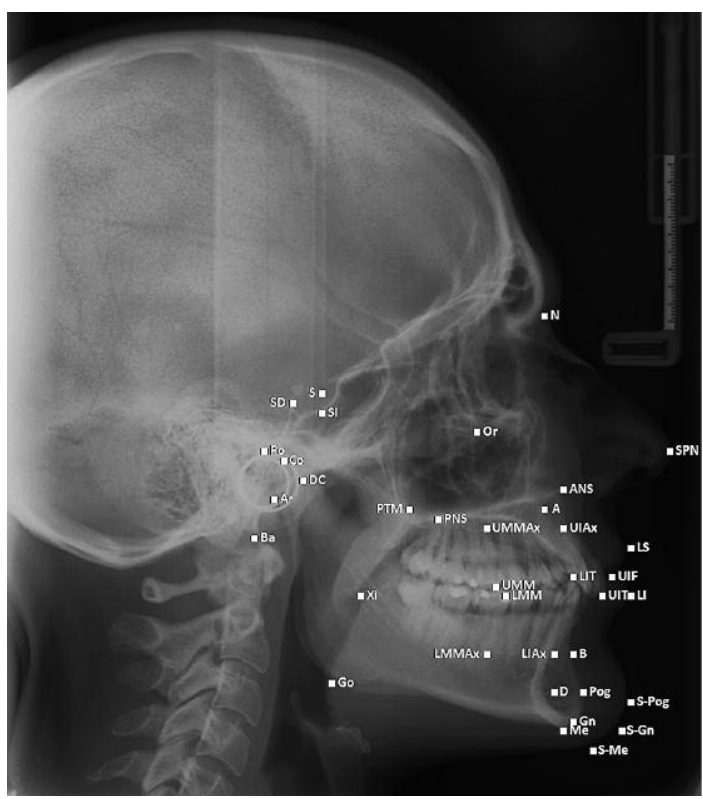

angle - sella-nasion-B point): class I (ANB angle $=2 \pm 2^{\circ}$ ), class II (ANB angle $>4^{\circ}$ ), and class III (ANB angle $\left\langle 0^{\circ}\right.$ ). The following parameters were studied: cranial base relationships: cranial base flexure, nasion-sella-basion angle (N-S-Ba angle), saddle angle, nasion-sella-articulare angle (N-S-Ar angle), sellanasion-pogonion angle (S-N-Pg angle); maxillomandibular skeletal relationships: sella-nasion-A point angle (SNA angle), sella-nasion-B point angle (SNB angle), ANB angle (difference between SNA and SNB angle), facial convexity (SN-Pg angle), pogonion-nasion-B point angle (Pog-NB angle); Wits appraisal (the difference between the projection of point $A$ and $B$ on the occlusal plane in $\mathrm{mm}$ ); articulare-gonion-menton angle (Ar-Go-Me angle); vertical skeletal relationships: Frankfurt-mandible-plane angle (FMA angle), SN (sella-nasion) to palatal plane angle (NL-NSL angle), SN to mandible plane angle (ML-NSL angle), maxilla-mandible plane angle (ML-

Figure 1. Landmarks for cephalometric analysis: S - sella: center of sella turcica; SD - dorsum sellae: most posterior point of sella turcica; $\mathrm{SI}$ - floor of sella; $\mathrm{N}$ - nasion: most anterior point of the nasofrontal suture; A point: deepest point on the mid-sagittal plane between anterior nasal spine and supradentale; B point: deepest point on the mid-sagittal plane between infradentale and pogonion; Pog - pogonion: most anterior point of the contour of the chin; Gn - gnathion: most anterior and inferior point on the contour of the chin; Me - menton: most inferior point on the contour of the mandibular symphysis; Go - gonion: most posterior and inferior point on the contour of the mandible angle; $\mathrm{Ar}$ - articulare: intersection of the sphenoid and posterior border of the condyle; Or - orbitale: lowermost point of the bony orbit; $\mathrm{Ba}$ - basion: lowest and most anterior point of the foramen magnum; UIT - upper incisor tip; UIAX - upper incisor apex; LIAx - lower incisor apex; LIT - lower incisor tip; LMM - lower molar mesial cusp; LMMAx - lower molar mesial apex; UMM - upper molar mesial apex; UMMAx - upper molar mesial cusp; Po - porion: most superior point of the external auditory meatus; ANS anterior nasal spine; PNS - posterior nasal spine; Co condylion: most superior and posterior point of the condylar head; DC - point: midpoint at the neck of the condyle; UIF - upper incisor facial: most anterior point on the facial surface of the upper incisor crown; LIF - lower incisor facial: most anterior point on the lower incisor crown; SPN - soft pronasale: the tip of the nose; LS - soft labrale sup.: the most anterior point of the upper lip; LI - soft labrale inf.: the most anterior point of the lower lip; S-Pog - soft pogonion; S-Gn - soft gnathion; S-Me - soft menton; Xi - point: a point at the center of the mandible ramus, opposite the mandible foramen; PTM: the lowest point of the contour of the pterygomaxillary fissure; D point: the center of the symphysis 
NL angle), occlusal plane to SN angle, lower anterior facial height/total anterior facial height (anterior nasal spine-menton: ANS-Me/N-Me\%); horizontal relationships: the constructed horizontal plane through the nasion at $7^{\circ}$ clockwise from the sella, measuring the distances projected on this plane: nasion-A, nasion-B, nasion-pogonion (NA-HP, NB-HP, $\mathrm{NPg}-\mathrm{HP} \mathrm{mm}$ ), the angle between the mandible plane and the horizontal plane (MP-HP angle), dental relationships: interincisal angle (angle between upper and lower incisor), maxillary incisor to NA plane angle, mandible incisor to NB plane angle, maxillary incisor to NA plane distance $(\mathrm{mm})$, mandible incisor to NB plane distance $(\mathrm{mm})$, overjet (sagittal distance between the incisor ridges of the upper incisor teeth labially or buccally to the incisor margins of the lower incisor teeth when the jaws are closed normally) ( $\mathrm{mm}$ ), overbite (vertical distance between the incisor ridges of the upper incisor and lower incisor margins when the jaws are closed normally) ( $\mathrm{mm})$, midline shift (distance between the maxillary and mandibular midlines when the jaws are closed normally) ( $\mathrm{mm}$ ), mandible incisor to mandible plane angle, soft tissue relationships: upper lip to E-line distance $(\mathrm{mm})$, lower lip to E-line distance ( $\mathrm{mm}$ ) (Figure 2).

\section{Statistical analysis}

The data obtained were statistically processed using Statistical Package for the Social Sciences (SPSS Inc., Chicago, USA, 13.0 version for Windows). To study the existence of significant differences of different quantitative features that follow a normal distribution, parametric Student tests with equal or unequal variations were used (variations were previously tested with the Levene test). The null hypothesis $(\mathrm{HO})$ was that the mean studied features of the experimental group (subjects with TMD) do not differ significantly from the control group (subjects without TMD) in two independent samples. Significance level we assumed to be equal with 0.05 . To study the existence of significant differences of averages between multiple groups, the Analysis of Variance (ANOVA) was used. If a significant test analysis was achieved, the Bonferroni correction was applied (with $\alpha=0.05 / 3=0.02$ ).

Existence and intensity and the correlation of quantitative variables overbite, overjet and SNB angle were studied by using the Pearson correlation coefficient and applying Colton's empirical rules.

\section{Results}

The subjects were grouped according to the ANB difference, into class I, II or III skeletal pattern, as an experimental group with TMD and a control group without TMD. The means of each measured category were calculated and significant differences between the experimental and control groups were examined by the $t$ test. According to the results from each class, no measurement showed significant sex differences between the experimental and the control group (Table II). The age distribution in class I was $20.73 \pm 3.99$ years in the experimental group, $23.70 \pm 4.47$ years in the control group; in class II it was $25.71 \pm 10.64$ years in the experimental group, and $23.68 \pm 3.22$ years in the control

Figure 2. Lines and angles used for the lateral cephalometric analyses. Constructed planes and lines: HP line, the constructed horizontal plane through the nasion at $7^{\circ}$ clockwise from sella, mandible plane (MP: Go-Gn line); palatal plane (anterior nasal spine - ANS - posterior nasal spine PNS line); occlusal plane; sella-nasion line (SN); Frankfurt horizontal (porion-orbitale: Po-Or); S-Ba (basion) line, S-Ar (articulare) line; N-Pg (pogonion) line; N-A line; N-B line; Ar-Go (gonion) line; upper incisor line (UIF-UIAx); lower incisor line (LIF-LIAx). Measured angles (NV - normal value): cranial base flexure (N-S-Ba angle, between NS line and S-Ba line, NV = $130 \pm 5^{\circ}$ ); saddle angle (N-S-Ar angle); facial convexity ( $\mathrm{SN}-\mathrm{Pg}$ angle, $\mathrm{NV}=75 \pm 4^{\circ}$ ); SNA angle $\left(N V=80 \pm 2^{\circ}\right)$; SNB angle $\left(N V=78 \pm 2^{\circ}\right)$; Pog$\mathrm{NB}$ angle $\left(\mathrm{NV}=2 \pm 2^{\circ}\right)$; Ar-Go-Me angle $\left(\mathrm{NV}=120 \pm 2^{\circ}\right)$; FMA angle $\left(\mathrm{NV}=25 \pm 3^{\circ}\right)$; mandible plane-HP angle (MP-HP angle, NV $=25 \pm 5^{\circ}$ ); mandible incisor to mandible plane angle $\left(\mathrm{NV}=88 \pm 3^{\circ}\right)$; $\mathrm{SN}$ to palatal plane angle (NL-NSL angle, NV $=8.5 \pm 3^{\circ}$ ); SN to mandible plane angle ( $\mathrm{ML}$-NSL angle, $\mathrm{NV}=32 \pm 6^{\circ}$ ); maxilla-mandibular plane angle $\left(M L-N L\right.$ angle, $\left.N V=23.5 \pm 3^{\circ}\right)$; interincisal angle $\left(\mathrm{NV}=131 \pm 6^{\circ}\right)$; maxillary incisor to NA plane angle $\left(N V=22 \pm 3^{\circ}\right)$; mandible incisor to NB plane angle $\left(\mathrm{NV}=25 \pm 3^{\circ}\right)$. Measured distances $(\mathrm{mm})$ : projected dis-

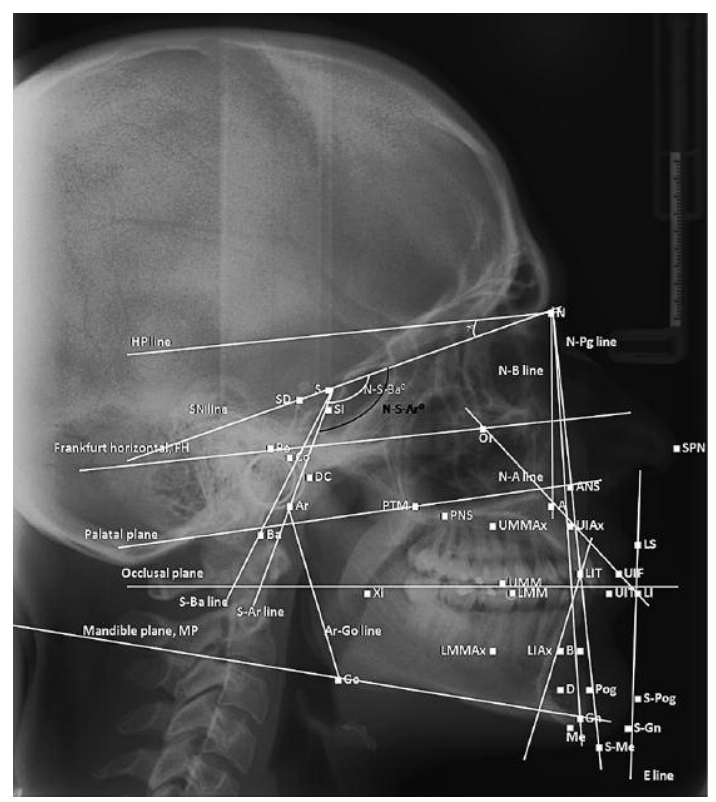
tances upon HP line: Ar-PTM, PTM-N, N-A, N-Pog, N-B; PNS-ANS; lower anterior height/total anterior facial height (\%); upper incisor-palatal plane; lower incisor-mandible plane; upper molar-palatal plane; lower molar mandible plane; Ar-Go; maxillary incisor-NA plane; mandible incisorNB plane; overjet ( $N V=2 \pm 2 \mathrm{~mm}$ ); overbite ( $N V=2 \pm 2 \mathrm{~mm}$ ); Wits appraisal ( $N V=2 \pm 2 \mathrm{~mm}$ ) 
Table II. Distribution of subjects according to sex

\begin{tabular}{|c|c|c|c|c|c|c|c|c|c|}
\hline \multirow[t]{2}{*}{ Sex } & \multicolumn{2}{|c|}{ Class I } & \multicolumn{2}{|c|}{ Class II } & \multicolumn{2}{|c|}{ Class III } & \multicolumn{2}{|c|}{ Pearson $\chi^{2}$ test } & \multirow{2}{*}{$\begin{array}{l}\text { Fisher's } \\
\text { exact test }\end{array}$} \\
\hline & Control & $\begin{array}{l}\text { Experi- } \\
\text { mental }\end{array}$ & Control & $\begin{array}{l}\text { Experi- } \\
\text { mental }\end{array}$ & Control & $\begin{array}{l}\text { Experi- } \\
\text { mental }\end{array}$ & Control & $\begin{array}{l}\text { Experi- } \\
\text { mental }\end{array}$ & \\
\hline Female & $74.40 \%$ & $28.60 \%$ & $72.70 \%$ & $27.30 \%$ & $28.60 \%$ & $71.40 \%$ & NS & NS & NS \\
\hline Male & $75 \%$ & $25.00 \%$ & $80 \%$ & $20 \%$ & $0 \%$ & $100 \%$ & NS & NS & NS \\
\hline
\end{tabular}

NS - not significant

group; in class III it was $27.40 \pm 7.46$ years in the experimental group, and $22.02 \pm 1.51$ years in the control group. $79.16 \%$ of the subjects had moderate TMD; $20.83 \%$ had severe TMD. Higher prevalence of TMD was found in females. In class I subjects, joint noises (83.33\%) followed by muscular pain $(16.66 \%)$ and TMJ pain (16.66\%) constituted the most frequent reported symptoms. In class II subjects, joint noises (71.42\%) followed by reduced mandible mobility (14.28\%), muscular pain (14.28\%) and TMJ pain (14.28\%) were the most frequent symptoms. In class III subjects, joint noises $(90.90 \%)$ followed by muscular pain ( $45.45 \%)$, reduced mandible mobility (27.27\%) and TMJ pain (27.27\%) were the most frequent symptoms. Angles and linear distances between the experimental and control groups from the class I, class II, and class III, grouped according to the sagittal skeletal relationship, were examined to observe more accurately the cephalometric characteristics of each sample. Table III shows the results of the cephalometric differences among the studied variables. Eleven measurements showed statistically significant differences. Among the statistically significant measurements, in class III patients the SNB angle and horizontal relationships were more significant than the other variables. In class II, the interincisal angle and overbite were more significantly modified in the experimental sample.

There was a good negative linear correlation between SNB angle and overbite in class I subjects $(r=-0.43, p=0.042)$ and between SNB angle and overjet ( $r=-0.60, p=0.002$ ). Wits appraisal was longer in the experimental group, when compared to the control group in all sagittal classes.

\section{Discussion}

This study was performed to establish the relationship between craniofacial skeletal structures and TMD in a group of subjects with malocclusions. There are reports on craniofacial structures in patients with TMD on lateral cephalograms by Brand et al. [26], Nebbe et al. [27-29], Simmons et al. [30], Sonnesen and Svensson [31], Ciancaglini et al. [32], Dibbets and van der Weele [33], and Sonnesen et al. [34]. Many studies have focused on the TMJ relationships of class II patients. This study focused on the relationships of craniofacial structures in class I, class II and class III subjects. It is important to find out if there is an underlying temporomandibular disorder before the beginning of any irreversible treatment. In this study, overjet was statistically higher in subjects with temporomandibular disorders, independently of the sagittal skeletal pattern. Therefore, an examination of the TMJ in subjects with a large overjet should be considered. This study did not reveal any differences between vertical skeletal relationships in subjects with sagittal malocclusions and TMJ disorder. Cranial base inclinations were found significant in class II subjects. Still, before relating this difference to the development of TMD, further studies with larger samples must be considered. There were not found significant differences between TMD and sex. No difference was noted between associated anomalies or specific types of malocclusions and signs and symptoms of TMD. Bakke suggests that even if much controversy has been reported regarding the role of occlusion in TMD, there is, however, no doubt that occlusal variables influence natural masticator muscle function [35]. This study reports a significant influence of overbite on signs of TMD. Thilander et al. [36] demonstrated that TMD is significantly associated with posterior crossbite, anterior open bite, Angle Class III malocclusion, and extreme maxillary overjet. The question of whether such measures will also prevent development of TMD or decrease the TMD signs in such patients is still open to discussion since the cause of mandible dysfunction is obviously multifactorial [36]. In this study no statistically significant changes were found within class I and III subjects concerning overbite, although it was steeper in the experimental group when compared to the control group of each class. Class II experimental subjects had a higher anterior open bite when compared to the control group, the difference being statistically significant. Dibbets and van der Weele [37], Egermark et al. [38], Helm and Petersen [39], and Sadowsky and Polson [40] failed to identify significant associations between malocclusion and TMD. Byun et al. suggested that patients with TMD can have clinical symptoms irrespective of any orthodontic treatment, as the TMJ has a normal adaptive capacity to withstand injuries and external factors contributing to the disturbance of condyle equilibrium. Temporomandibular disorders include several clinical conditions that are pain-related; the 


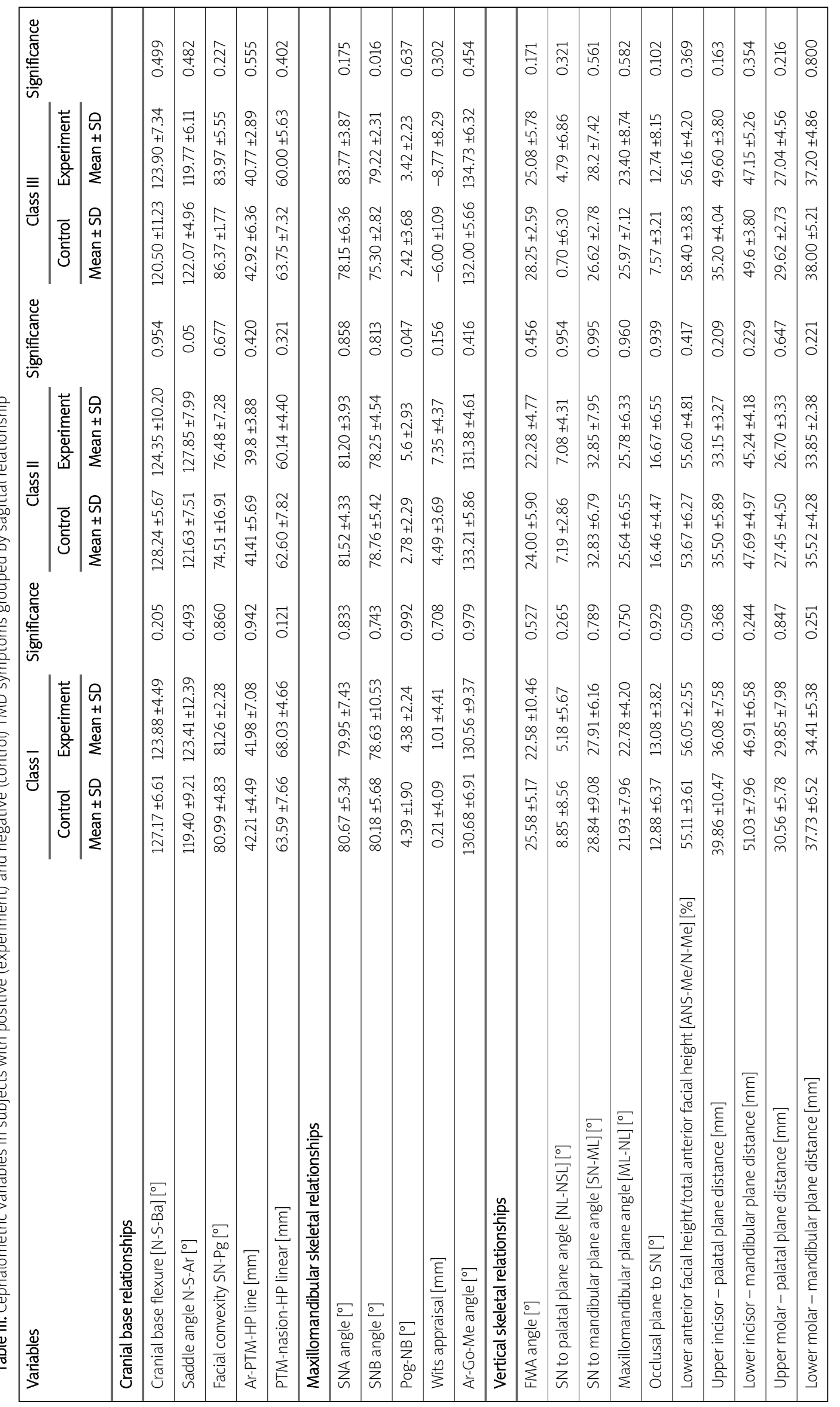




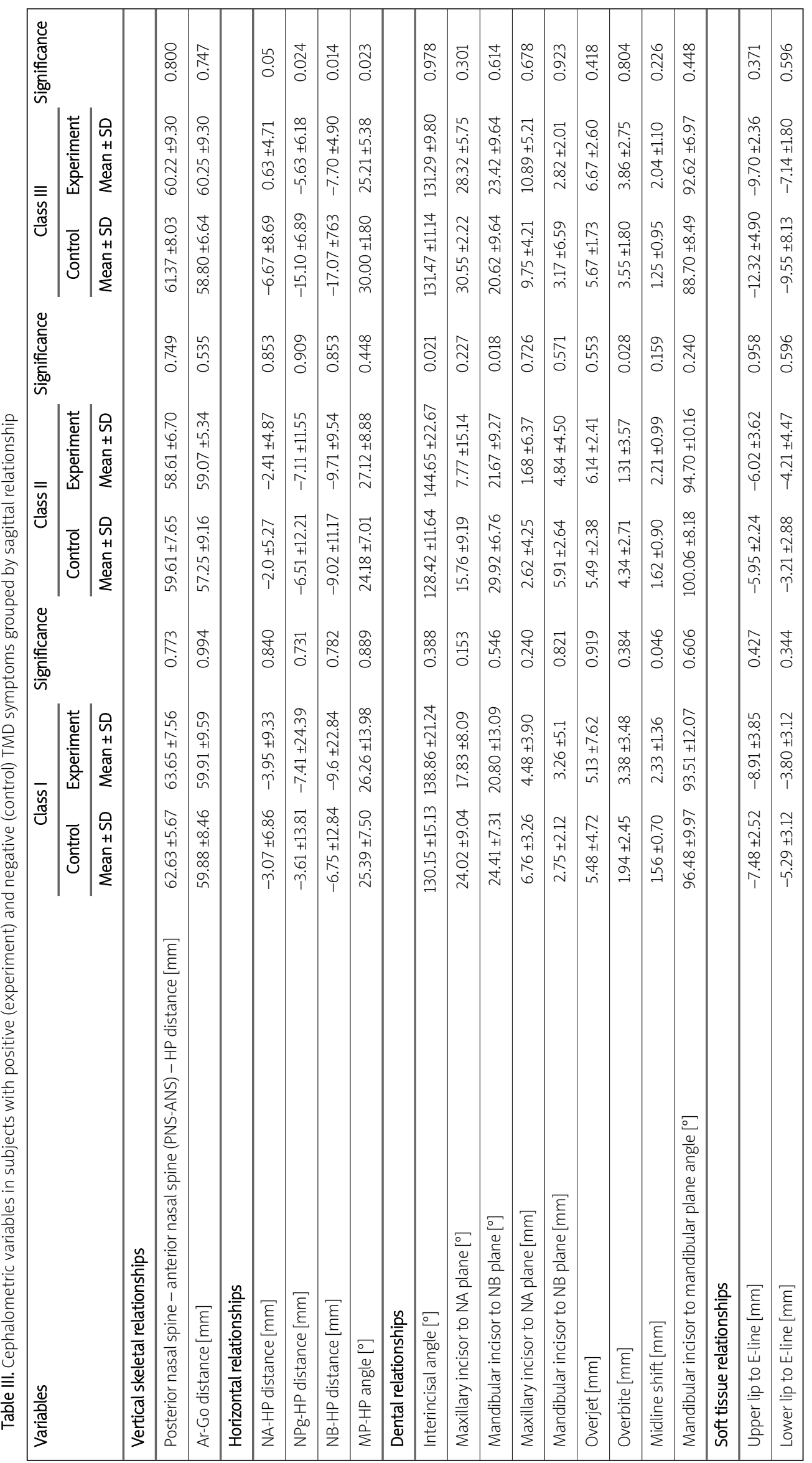


origin of pain in the craniofacial region is not yet fully understood, but is known to be multifactorial, as shown by Schulte et al. [41]. Pain is perceived differently by subjects, whether it is muscle, joint or acute postoperative pain. The objective evaluation of pain intensity is a long-term problem, pain being perceived by the organism as a strong stimulus which evokes trigger reactions such as sensitization, nociception, or neurohumoral modulation [42].

Regardless of the cause-effect relationship, it is important to screen out patients with TMD before beginning any irreversible treatment, including orthodontic treatment, because TMJ disorder positive signs and symptoms during or after treatment might be interpreted to be a result of the treatment [43]. The present study has tried to detail specific signs of temporomandibular alteration and to assess the relationship between craniofacial skeletal structures and temporomandibular disorders. Chung-Ju et al. reported that there is much controversy about the relationship between TMJ disorders and the skeletal structures of the lower face. It is not clear whether the disharmony of the facial skeletal structure is caused by the TMJ disorder or vice versa. A TMJ disorder has a close relationship with lower facial structure, which must be considered during treatment planning [20]. An analysis of the craniofacial structure with lateral cephalograms showed their further use in diagnosis and treatment planning for TMJ disorder patients. Reynders [44], Tallents et al. [45], Vanderas [46], and Henrikson [47] consider that the question of whether the occurrence of malocclusion traits are related to signs and symptoms of TMD has attracted considerable interest.

It is of great value to examine the TMJ of a patient with malocclusion, to prevent any misrelated signs and symptoms of the TMD. Although lateral cephalograms do not assess the temporomandibular joint, they offer sufficient relevant information regarding skeletal pattern; orientating specialists in assessing the structural dimensions of the skeletal pattern and bringing new elements in diagnosing a temporomandibular disorder. When observing large overjet, midline shift and deep bite, one should also consider a TMJ evaluation, there being an evident association between TMJ and skeletal pattern. In this study there has been evidenced a correlation between structure of the lower face, characteristics of the facial profile and the temporomandibular disorder. TMD signs and symptoms fluctuate unpredictably, indicating an increased demand for evaluating TMJ of patients with malocclusions. As stated by Michalak et al., along with establishing the type of malocclusion it is important to determine the occurence of parafunctions, as they can lead to changes in the temporomandibular joint [48].
In conclusion, the TMJ status is an important factor to consider in subjects with malocclusions. Midline shift, large overjet and deep overbite have been associated with signs and symptoms of temporomandibular disorders. Joint noises followed by reduced mandible mobility, muscular pain and joint pain were the most frequent symptoms in subjects with TMJ disorders.

\section{Acknowledgments}

This study is supported by funding from a POSDRU/ 107/1.5/S/78702 project from the University of Medicine and Pharmacy "Iuliu Hațieganu" Cluj-Napoca, Romania.

\section{References}

1. Okeson JP. Orofacial pain. Guidelines for assessment, diagnosis and management. Quintessence Pub, Chicago 1996; 113-84.

2. Mohlin B, Ingervall B, Thilnader B. Relationship between malocclusion and mandibular dysfunction in Swedish men. Eur J Orthod 1980; 2: 229-38.

3. Sonnesen L, Bakke M, Solow B. Malocclusion traits and symptoms and signs of temporomandibular disorders in children with severe malocclusions. Eur J Orthod 1998; 19: 543-59.

4. Alamoudi N. The correlation between occlusal characteristics and temporomandibular dysfunction in Saudi Arabian children. J Clin Pediatr Dent 2000; 24: 229-36.

5. Ali IM, Yamada K, Alkhamrah B, Vergara R, Hanada K. Relationship between occlusal curvatures and mandibular deviation in orthodontic patients with temporomandibular disorders. J Oral Rehabil 2003; 30: 1095-103.

6. Gesch D, Bernhardt O, Alte D, Kocher T, John U, Hensel E. Malocclusions and clinical signs or subjective symptoms of temporomandibular disorders (TMD) in adults. Results of the population based Study of Health in Pomerania (SHIP). J Orofac Orthoped 2004; 65: 88-103.

7. Henrikson T, Ekberg EC, Nilner M. Symptoms and signs of temporomandibular disorders in girls with normal occlusion and class II malocclusion. Acta Odontol Scand 1997; 55: 229-35.

8. Kahn J, Tallents RH, Katzberg RW, Ross ME, Murphy WC. Prevalence of dental occlusal variables and intraarticular temporomandibular disorders: molar relationship, lateral guidance, and nonworking side contacts. J Prost Dent 1999; 82: 410-5.

9. Liu ZJ, Yamagata K, Kuroe K. Morphological and positional assessments of TMJ components and lateral pterygoid muscle in relation to symptoms and occlusion of patients with temporomandibular disorders. J Oral Rehabil 2000; 27: 860-74

10. Manfredini D, Landi N, Romagnoli M, Bosco M. Psychic and occlusal factors in bruxers. Aust Dent J 2004; 49: 84-9.

11. Tuerlings $\vee$, Limme $M$. The prevalence of temporomandibular joint dysfunction in the mixed dentition. Eur J Orthod 2004; 26: 311-20.

12. Yatani H, Minakuchi H, Matsuka Y, Fujisawa T, Yamashita A. The long term effect of occlusal therapy on self-administered treatment outcomes of TMD. J Orofac Pain 1998; 12: 75-88.

13. Pullinger AG, Seligman DA, Gornbein JA. A multiple logistic regression analysis of the risk and relative odds of tem- 
poromandibular disorders as a function of common occlusal features. J Dent Res 1993; 72: 968-79.

14. Riolo ML, Brandt D, Have TR. Associations between occlusal characteristics and signs and symptoms of TMJ dysfunction in children and young adults. Am J Orthod Dentofacial Orthop 1987; 92: 467-77.

15. Abrahamssona C, Ekbergb EC, Henriksonc T, Nilnerd M, Sunzele B, Bondemarkf L. TMD in consecutive patients referred for orthognathic surgery. Angle Orthod 2009; 79: 621-7.

16. Michelotti A, lodice G. The role of orthodontics in temporomandibular disorders. J Oral Rehab 2010; 37: 411-29.

17. Gremillion HA. The relationship between occlusion and TMD: an evidence-based discussion. J Evid Based Dent Pract 2006; 6: 43-7.

18. Kalamir A, Pollard H, Vitiello AL, Bonello R. TMD and the problem of bruxism. A review. J Body Mov Ther 2007; 11: 183-93.

19. Yu S, Xing X, Liang S, et al. Locally synthesized estrogen plays an important role in the development of TMD. Med Hypotheses 2009; 72: 720-2.

20. Chung-Ju H, Sang-Jin S, Suk-Joo K. Lateral cephalometric characteristics of malocclusion patients with temporomandibular joint disorder symptoms. Am J Orthod Dentofacial Orthop 2006; 129: 497-503.

21. Athanasiou AE, Melsen B. Craniomandibular dysfunction following surgical correction of mandibular prognatism. Angle Orthod 1992; 62: 9-14.

22. Steiner CC. Cephalometrics for you and me. Am J Orthod 1953; 39: 729-55

23. Hasund A, Boe O, Jenatsche F, Norderval K, Thunold K, Wisth PJ. Clinical cephalometry for the Bergen - Technique. University of Bergen, Norway 1974.

24. Segner D, Hasund A. Individualisierte Kephalometrie. 2. Aufage, Dietmar Segner Verlag und Vertrieb, Hamburg 1994.

25. Scheideman GB, Bell WH, Legan HL, Finn RA, Reisch JS. Cephalometric analysis of dentofacial normals. Am J Orthod 1980; 78: 404-20.

26. Brand JW, Nielson KJ, Tallents RH, Nanda RS, Currier GF, Owen WL. Lateral cephalometric analysis of skeletal patterns in patients with and without internal derangement of the temporomandibular joint. Am J Orthod Dentofacial Orthop 1995; 107: 121-8.

27. Nebbe B, Major PW, Prasad NG. Adolescent female craniofacial morphology associated with advanced bilateral TMJ disk displacement. Eur J Orthod 1998; 20: 701-12.

28. Nebbe B, Major PW. Prevalence of TMJ disk displacement in a pre-orthodontic adolescent sample. Angle Orthod 2000; 70: 454-63.

29. Nebbe B, Major PW, Prasad N. Female adolescent facial pattern associated with TMJ disk displacement and reduc tion in disk length: part I. Am J Orthod Dentofacial Orthop 1999; 116: 168-76.

30. Simmons HC 3rd, Oxford DE, Hill MD. The prevalence of skeletal Class II patients found in a consecutive popula tion presenting for TMD treatment compared to the national average. J Tenn Dent Assoc 2008; 88: 16-8.

31. Sonnesen L, Svensson P. Temporomandibular disorders and psychological status in adult patients with a deep bite. Eu J Orthod 2008; 30: 621-9.

32. Ciancaglini R, Colombo-Bolla G, Gherlone EF, Radaelli G. Orientation of craniofacial planes and temporomandibular disorder in young adults with normal occlusion. J Oral Rehabil 2003; 30: 878-86.

33. Dibbets JM, van der Weele LT. Signs and symptoms of temporomandibular disorder (TMD) and craniofacial form. Am J Orthod Dentofacial Orthop 1996; 110: 73-8.
34. Sonnesen L, Bakke M, Solow B. Temporomandibular dis orders in relation to craniofacial dimensions, head posture and bite force in children selected for orthodontic treatment. Eur J Orthod 2001; 23: 179-92.

35. Bakke M. Mandibular elevator muscles: physiology, action, and effect of dental occlusion. Scand J Dent Res 1993; 101: 314-31.

36. Thilander B, Rubio G, Pena L, de Mayorga C. Prevalence of temporomandibular dysfunction and its association with malocclusion in children and adolescents: an epidemiologic study related to specified stages of dental development. Angle Orthod 2002; 72: 146-54.

37. Dibbets JM, van der Weele LT. Orthodontic treatment in relation to symptoms attributed to dysfunction of the temporomandibular joint. A 10-year report of the University of Groningen study. Am J Orthod Dentofacial Orthop 1987: 91: 193-9.

38. Egermark I, Magnusson T, Carlsson GE. A 20-year followup of signs and symptoms of temporomandibular disorders and malocclusions in subjects with and without orthodontic treatment in childhood. Angle Orthod 2003; 73 109-15.

39. Helm S, Petersen PE. Mandibular dysfunction in adulthood in relation to morphologic malocclusion at adolescence. Acta Odontol Scand 1989; 47: 307-14.

40. Sadowsky C, Polson AM. Temporomandibular disorders and functional occlusion after orthodontic treatment: result of two long-term studies. Am J Orthod 1984; 86: 386-90.

41. Schulte F, Filis A, Karageorgos N, Sandu N, Schaller B. Pain after craniotomy - really a problem? Arch Med Sci 2010; 6: 827-8.

42. Fricova J, Vejražka M, Stopka P, Krizova J, Běláček J, Rokyta $R$. The influence of pre-emptive analgesia on postoperative analgesia and its objective evaluation. Arch Med Sci 2010; 6: 764-71.

43. Byun ES, Ahn SJ, Kim TW. Relationship between internal derangement of the temporomandibular joint and dentofacial morphology in women with anterior open bite. Am J Orthod Dentofacial Orthop 2005; 128: 87-95.

44. Reynders RM. Orthodontics and temporomandibular disorders: a review of the literature (1966-1988). Am J Orthod Dentofacial Orthop 1990; 97: 463-71.

45. Tallents RH, Catania J, Sommers E. Temporomandibular joint fi ndings in pediatric populations and young adults: a critical review. Angle Orthod 1991; 61: 7-16.

46. Vanderas AP. Relationship between malocclusion and craniomandibular dysfunction in children and adolescents: a review. Pediatric Dentistry 1993; 15: 317-22.

47. Henrikson T. Temporomandibular disorders and mandibular function in relation to Class II malocclusion and orthodontic treatment. Swedish Dent J 1999; 23 (Suppl 134): 1-144.

48. Michalak M, Wysokińska-Miszczuk J, Wilczak M, Paulo M, Bożyk A, Borowicz J. Correlation between eye and ear symptoms and lack of teeth, bruxism and other parafunctions in a population of 1006 patients in 2003-2008. Arch Med Sci 2012; 8: 104-10. 